\title{
Controlling in Industrie und Dienstleistung im Mittelpunkt
}

\author{
Bei der $9^{\text {th }}$ Manufacturing Accounting Research Conference in Münster diskutieren \\ Wissenschaftler aus aller Welt aktuelle Fragen des Controllings
}

Kostenmanagement in Zusammenarbeit mit Lieferanten und Netzwerkpartnern, neue Ansätze zur Budgetierung und zum Performance Measurement, Implementierung neuer Controllingsysteme, Einflüsse der Internationalisierung auf das Controlling - diese und viele andere Themen, die das Controlling in Industrie- und Dienstleistungsunternehmen bewegen, stehen im Mittelpunkt der $9^{\text {th }}$ Manufacturing Accounting Research Conference, die vom 21. bis 24. Juni 2009 in Münster stattfindet.

Die Konferenz wird vom Lehrstuhl für Internationale Unternehmensrechnung an der Westfälischen Wilhelms-Universität Münster (Prof. Dr. Peter Kajüter) in Kooperation mit dem European Institute for Advanced Studies in Management (EIASM) vom 21. bis 24. Juni 2009 ausgerichtet. Sie führt eine im Jahre 1993 begonnene internationale Konferenzreihe fort, die alle zwei Jahre in einer anderen europäischen Stadt stattfindet.

Auf der diesjährigen Konferenz werden u. a. Professor Alnoor Bhimani (London School of Economics and Political Science), Professor Falconer Mitchell (University of Edinburgh) und Professor Ken Merchant (University of Southern California) als Key Note Speaker über neueste Trends im Controlling referieren. Eine Podiumsdiskussion mit Vertretern aus Wissenschaft, Beratungs- und Unternehmenspraxis wird sich aus unterschiedlichen Perspektiven der Frage widmen, inwieweit die Controllingforschung nützliche Ergebnisse für die Praxis liefert. Das Conference Dinner bietet anschließend die Möglichkeit zum weiteren Erfahrungsaustausch zwischen Wissenschaft und Praxis.
Für Wissenschaftler besteht die Gelegenheit, im Rahmen der Tagung aktuelle Forschungsergebnisse zu präsentieren und zu diskutieren. Für Doktoranden wird eine spezielle Plattform geboten, um Feedback zu ihren Arbeiten zu erhalten. Deadline für die Einreichung von Forschungspapieren ist der 15. März 2009.

Weitere Informationen zu dieser

Konferenz erhalten Sie unter:

http://www.wiwi.uni-muenster.de/iur

Westfälische Wilhelms-Universität

Münster

Lehrstuhl für BWL, insb.

Internationale Unternehmensrechnung

Prof. Dr. Peter Kajüter

Universitätsstraße 14 - 16

48143 Münster

Tel.: 02 51 / 83 - 22840

E-Mail.: iur@wiwi.uni-muenster.de 